\title{
ON RINGS WITH A HIGHER DERIVATION
}

\author{
SHIZUKA SATO
}

Abstract. Let $R \supset \mathcal{O}$ be two rings with the unit 1 . Then we set $R(\Theta, R)=\left\{x \in R ; x^{r} \in \mathcal{O}\right.$ for some integer $\left.r \geqq 1\right\}$. At first, it is shown that, under some assumptions, $d \Theta \subset \mathcal{O}$ implies $d R(\Theta, R)$ $\mathcal{C R}(\Theta, R)$. Next, with the Lying-over Theorem on $d$-differential ideals, we show: Let $(R, M)$ and $(\Theta, m)$ be two quasi-local rings and let $d$ be a higher derivation of rank $\infty$ of the total quotient ring of $R$ such that $d \theta \subset \theta$. Suppose that $R$ is integral over $\theta$ and $\theta$ is dominated by $R$. Then $d(m) \subset m$ implies $d(M) \subset M$.

0 . Terminology. In this paper, we assume that all rings are commutative and have the unit 1. Let $R \supset 0$ be two rings. Then we set:

$$
\begin{gathered}
\mathbb{R}(\mathcal{O}, R)=\left\{x \in R ; x^{r} \in \mathcal{O} \text { for some integer } r \geqq 1\right\}, \\
\mathbb{R}(\mathcal{O}, R)^{*}=\{x \in R ; \exists y \in \mathcal{O} \text { such that } y x \in \mathbb{R}(\mathcal{O}, R)\} .
\end{gathered}
$$

Next, let $R^{(s)}$ be the set of $s$-tuples $\left(\rho_{0}, \rho_{1}, \cdots, \rho_{s-1}\right), \rho_{i} \in R$, with operations, for $x=\left(x_{0}, x_{1}, \cdots, x_{s-1}\right), y=\left(y_{0}, y_{1}, \cdots, y_{8-1}\right) \in R^{(s)}$,

$$
\begin{aligned}
x+y & =\left(x_{0}+y_{0}, x_{1}+y_{1}, \cdots, x_{s-1}+y_{s-1}\right), \\
x y & =\left(z_{0}, z_{1}, \cdots, z_{s-1}\right), \quad \text { where } z_{k}=\sum_{i+j=k} x_{i} y_{j} .
\end{aligned}
$$

This $R^{(s)}$ is a ring and $R^{(\infty)}$ is isomorphic to a formal power series ring $R[[t]]$ with one indeterminate $t$ over $R$.

1. On the existence theorems. Let $d=\left(d_{i}\right)_{0 \leq_{i \leq s-1}}$ be a higher derivation of rank $s$ of $R$ in the sense of P. Ribenboim (cf. [1]). Let $\mathfrak{A}$ be an ideal of a ring $R$. Then we shall call $\mathfrak{A}$ a $d$-differential ideal if $d \mathfrak{A}$ $\subset \mathfrak{A}$, where $d \mathfrak{U} \subset \mathfrak{U}$ means $d_{i} \mathfrak{U} \subset \mathfrak{U}$ for all $i$.

THEOREM 1. Let d be a higher derivation of rank $s$ (finite or infinite) of $R$. If $\mathfrak{A}$ is a d-differential ideal of $R$, then there exists a maximal $d$ differential ideal $\mathfrak{M}$ of $R$ such that $\mathfrak{M} \supset \mathfrak{A}$.

Proof. Let $\mathfrak{F}$ be the set of $d$-differential ideals containing $\mathfrak{A}$. Then $\mathfrak{F}$ is an inductive set (the order being given by the inclusion relation). Hence Zorn's Lemma implies the existence of $\mathfrak{M}$.

The following proposition is almost the same as Theorem 1 in [3]. But the difference between the two propositions is the definition of higher derivations.

Received by the editors November 12, 1970.

AMS 1970 subject classifications. Primary $16 \mathrm{~A} 72$.

Key words and phrases. Higher derivations, $d$-differential ideals, quasi-local rings. 
Proposition 1. Let $d=\left(d_{i}\right)$ be a higher derivation of a Noetherian ring $\mathcal{O}$ such that $d_{0}$ is an isomorphism. Let $\mathfrak{A}$ be a d-differential ideal of $\mathcal{O}$, and let $z_{1}, z_{2}, \cdots, z_{r}$ be associated prime ideals of $\mathfrak{A}$. Then $z_{1}$, $\jmath_{2}, \cdots, \jmath_{r}$ are d-differential ideals and $\mathfrak{A}$ can be written as an irredundant intersection of d-differential primary ideals.

Proof. We can prove this proposition by almost the same way as Theorem 1 of [3], by considering $\exp (d), \exp \left(d^{\prime}\right)$ instead of $\exp (t D)$, $\exp (-t D)$ in $[3]$ respectively, where $d^{\prime}$ is a higher derivation such that $d d^{\prime}=1$.

Corollary. With the same $\mathcal{O}, \mathfrak{A}$ and $d=\left(d_{i}\right)$ as Proposition 1 , there exists always a maximal d-differential ideal of $\mathcal{O}$ containing $\mathfrak{A}$ and this is a prime ideal.

TheORem 2. Let $R \supset \mathcal{O}$ be two rings and let $d=\left(1, d_{1}, d_{2}, \cdots\right)$ be a higher derivation of the total quotient ring of $R$ such that dOCO and $d R \subset R$. Assume that $R$ is integral over $\mathcal{O}$. If $z$ is a d-differential prime ideal of $\mathcal{O}$, then there exists a d-differential prime ideal $z^{\prime}$ of $R$ such that $z^{\prime} \cap \mathcal{O}=z$.

Proof. First, we shall prove that it is sufficient to consider the case when $\mathcal{\theta}$ is an $d$-differential quasi-local ring and the maximal ideal of $\mathcal{\theta}$ is $d$-differential. By the hypothesis, $d\left(\mathcal{O}_{z}\right) \subset \mathcal{O}_{3}$. On the other hand, for $s \in \mathcal{O}-z, \quad d_{l}(1 / s)=f(s) / s^{l+1}, f(s) \in \mathcal{O}$. Since $d_{z} \subset_{z}, \quad d\left(z_{3} \mathcal{O}_{z} \subset_{z} \Theta_{z}\right.$. Hence $z \Theta_{z}$ is a $d$-differential ideal and is the maximal ideal of $\mathcal{O}_{3}$. Let $S=\mathcal{O}-\mathfrak{z}$. Then $R_{s}$ is integral over $\mathcal{O}_{s}$. Now, suppose that there is a $d$ differential prime ideal $\mathfrak{M}^{\prime}$ of $R_{8}$ such that $\mathfrak{M}^{\prime} \cap \Theta_{8}={ }_{z} \Theta_{8}$. Let $\pi$ be the natural mapping $\pi(a)=a \cdot 1$ of $\mathcal{O}$ into $\mathcal{O}_{s}$. Then $z^{\prime}=\pi^{-1}\left(\mathfrak{M}^{\prime}\right)$ is a $d$ differential prime ideal. For let $x \in z^{\prime}$ and $\pi(x)=y \in \mathfrak{M}^{\prime}$. Then, $\pi\left(d_{i}(x)\right)=d_{i}(x) \cdot 1=d_{i}((x) \cdot 1)=d_{i}(\pi(x))$ and $\pi(x) \in \mathfrak{M}^{\prime}$. Hence $d_{i}(\pi(x))$ $\in \mathfrak{M}^{\prime}$ and $d_{i}(x) \in z^{\prime}$. Trivially, $z^{\prime} \cap \mathcal{O} \supseteq$. Conversely, let $x \in z^{\prime} \cap \mathcal{O}$ and $\mathfrak{N}=\operatorname{Ker}(\pi)$. Further let $\pi^{*}: \mathcal{\Theta} \rightarrow \mathcal{O} / \mathfrak{N}$ be the canonical mapping. $\mathcal{O} / \mathfrak{N}$ is isomorphic to a subring of $\mathcal{\Theta}_{s}$. Hence $\pi^{*}(x)$ is considered as an element of $\mathcal{O}_{8}$. Therefore $\pi^{*}(x) \in \mathfrak{N}^{\prime} \cap \mathcal{O}_{8}$, and $x \in \in_{z} \mathcal{O}_{8}$. So, we have $x \in z$ and $z^{\prime} \cap \mathcal{\theta}=z$. Thus we may assume that $\theta$ is a quasi-local ring with a higher derivation $d$ and $z$ is the maximal ideal of $\theta$. It is well known that in this case $R_{z} \neq R$. Thus, by the Corollary of Proposition 1 , there is a maximal $d$-differential ideal $\mathfrak{M}$ of $R$ such that $\mathfrak{M} \supset R_{\mathfrak{z}}$, and $\mathfrak{M} \cap \mathcal{O}=z$.

2. On the invariability concerned with a higher derivation. Let $d=\left(d_{i}\right)$ be a higher derivation of rank $s$ (finite or infinite) of $R$. Then we introduce the ring homomorphism $\exp (d)$ of $R^{(s+1)}$ in to $R^{(s+1)}$ as: 


$$
\text { for } x=\left(x_{i}\right) \in R^{(8+1)}, \quad \exp (d)(x)=\left(z_{k}\right) \quad \text { where } z_{k}=\sum_{i+j=k} d_{i}\left(x_{j}\right) .
$$

If $d_{0}$ is an isomorphism, then $d$ has inverse higher derivation $\delta$ of rank $s$ of $R$, i.e. $d \delta=1, \delta d=1$, where $d \delta=\left(d_{i}\right)\left(\delta_{j}\right)=\left(\epsilon_{k}\right), \epsilon_{k}=\sum_{i+j=k} d_{i} \delta_{j}$. Further,

$$
\exp (d \delta)=\exp (d) \exp (\delta)=1, \quad \exp (\delta d)=\exp (\delta) \exp (d)=1 .
$$

Hence $\exp (d)$ is an isomorphism [1].

First, we extend a theorem first proved by A. Seidenberg [2, Theorem 1] to the case of a higher derivation in a sense of P. Ribenboim [1].

PROPOSITION 2. Let $R$ つO be two rings and let $\mathcal{O}^{\prime}$ be the quasi-integral closure (or the complete integral closure) of $\theta$ in $R$. If $d$ is a higher derivation of rank $\infty$ of $R$ such that $d \mathcal{O} \subset \mathcal{O}$, then $d \mathcal{O}^{\prime} \subset \mathcal{O}^{\prime}$.

Proof. $R$ is considered as a subring of $R^{(\infty)}$ by the mapping: $x \rightarrow(x, 0,0, \cdots)$. Let $\alpha$ be an element of $\mathcal{O}^{\prime}$. Then there is an element $\beta \in \mathcal{O}$ such that $\beta \alpha^{\rho} \in \mathcal{O}$ for all $\rho \geqq 0$. Now, because $\exp (d)(\mathcal{O}) \subset \mathcal{O}^{(\infty)}$,

$$
\exp (d)\left(\beta \alpha^{\rho}\right)=\exp (d)(\beta)[\exp (d)(\alpha)]^{\rho} \in \mathcal{O}^{(\infty)} \text { for all } \rho \geqq 0 .
$$

Hence $d_{0}(\beta) d_{0}(\alpha)^{\rho} \in \mathcal{O}$ for all $\rho \geqq 0$. On the other hand, $d_{0}(\beta) \in \mathcal{O}$. Thus, we see that $d_{0}(\alpha) \in \mathcal{O}^{\prime}$. Assume that $d_{i}(\alpha) \in \mathcal{O}^{\prime}$ for $i \leqq N-1$. Then,

$$
\begin{aligned}
d_{0}(\beta)^{N} \exp (d)(\beta)[\exp (d)(\alpha) & \left.-\left(d_{0}(\alpha), \cdots, d_{N-1}(\alpha), 0,0, \cdots\right)\right]^{\rho} \\
= & \left(0, \underset{\rho_{N-1}}{\cdots}, 0, d_{0}(\beta)^{N+1} d_{N}(\alpha)^{\rho}, \cdots\right) \in \mathcal{O}^{(\infty)} .
\end{aligned}
$$

Therefore, by the induction assumption, $d_{0}(\beta)^{N+1} d_{N}(\alpha)^{\rho} \in \mathcal{O}$ for all $\rho \geqq 0$, and $d_{N}(\alpha) \in \mathcal{O}^{\prime}$. This completes the proof.

Next, we shall study the relation of $R(\mathcal{O}, R)$ and a higher derivation.

THEOREM 3. Let $k$ be a field of characteristic 0 and let $d=\left(d_{i}\right)$ be a higher derivation of rank $\infty$ of a domain $R(\supset k)$ such that $d_{0}$ is an isomorphism. Then $d k \subset k$ implies $d(R(k, R)) \subset R(k, R)$.

Proof. $d$ can be extended to a higher derivation of $K$ ( $=$ the quotient field of $R$ ). We shall denote by the same $d$ this extended higher derivation. Let $0 \neq x \in R(k, R)$. Then there is an integer $r \geqq 1$ such that $x^{r} \in k$. By the assumption, $d_{i}\left(x^{r}\right) \in k$ for all $i$. Hence, $\exp (d)\left(x^{r}\right)$ $=[\exp (d)(x)]^{r} \in k^{(\infty)}$. Thus $d_{0}(x)^{r} \in k$ and $d_{0}(x) \in R(k, R)$. Now, 


$$
\begin{aligned}
\exp (d)\left(x^{r}\right)- & \left(d_{0}(x), 0,0, \cdots\right)^{r} \\
& =\left(0, d_{1}(x), \cdots\right)\left(r d_{0}(x)^{r-1}, \cdots\right) \in k^{(\infty)} .
\end{aligned}
$$

Hence $r d_{0}(x)^{r-1} d_{1}(x) \in k$. As $d_{0}$ is an isomorphism and $d_{0}(x) \neq 0$, $d_{0}(x)^{-1} \in K$. Further, $d_{0}(x)^{r} \in k$ and $\left[d_{0}(x)^{r}\right]^{-1} \in k$. Thus $d_{0}(x)^{-1}$ $=d_{0}(x)^{r-1}\left[d_{0}(x)^{r}\right]^{-1} \in R$. Therefore $d_{0}(x)^{-1} \in R(k, R)$. Assume that $d_{i}(x) \in R(k, R), i \leqq N-1$. Then,

$$
\begin{aligned}
\exp (d)\left(x^{r}\right)- & \left(d_{0}(x), d_{1}(x), \cdots, d_{N-1}(x), 0,0, \cdots\right)^{r} \\
& =\left(0, \cdots, 0, r d_{0}(x)^{r-1} d_{N}(x), \cdots\right) \in R(k, R)^{(\infty)} .
\end{aligned}
$$

Hence $r d_{0}(x)^{r-1} d_{N}(x) \in R(k, R)$ and $d_{N}(x) \in R(k, R)$. This completes the proof of our assertion $d(R(k, R)) \subset R(k, R)$.

CoROLlaRY. Under the assumptions of Theorem $3, R(k, R)$ is a field.

TheOREM 4. Let $R$ つO be two domains and let $d=\left(d_{i}\right)$ be a higher derivation of rank $\infty$ of $R$. If $d \mathcal{O} \subset \mathcal{O}$ and $d(R(\mathcal{O}, R)) \subset R(\mathcal{O}, R)$, then $d\left(R(\mathcal{O}, R)^{*}\right) \subset \mathbb{R}(\mathcal{O}, R)^{*}$.

Proof. Let $x \in R(\mathcal{O}, R)^{*}$. Then there is an element $y \in \mathcal{O}$ such that $y x \in R(\mathcal{O}, R)$. By the hypothesis, $d_{0}(y x)=d_{0}(y) d_{0}(x) \in R(\mathcal{O}, R)$ and $d_{0}(y) \in \mathcal{O}$. Hence $d_{0}(x) \in R(\mathcal{O}, R)^{*}$. Now, $d_{0}(y) d_{1}(y x)=d_{0}(y)^{2} d_{1}(x)$ $+d_{0}(y) d_{0}(x) d_{1}(y), d_{0}(y) d_{1}(y x) \in \Re(\mathcal{O}, R)$ and $d_{0}(y) d_{0}(x) d_{1}(y) \in \Re(\mathcal{O}, R)$. Therefore $d_{0}(y)^{2} d_{1}(x) \in R(\mathcal{O}, R)$ and $d_{1}(x) \in R(\mathcal{O}, R)^{*}$. Assume that $d_{i}(x) \in R(\mathcal{O}, R)^{*}$ and $d_{i}(x) d_{0}(y)^{r+1} \in \mathcal{Q}(\mathcal{O}, R)$ for all $i \leqq N-1$. We have

$$
d_{0}(y)^{N} d_{N}(y x)=d_{0}(y)^{N+1} d_{N}(x)+d_{0}(y)^{N} \sum_{i+j=N ; i \geqq 1} d_{i}(y) d_{j}(x) .
$$

Hence $d_{0}(y)^{N+1} d_{N}(x) \in R(\mathcal{O}, R)$, by the induction assumption and the fact that $d_{N}(x) \in \mathcal{R}(\mathcal{O}, R)^{*}$. Thus we have $d\left(R(\mathcal{O}, R)^{*}\right) \subset \mathcal{R}(\mathcal{O}, R)^{*}$.

CorollaRy 1. Let $R \supset \mathcal{O}$ be two rings and let $d=\left(d_{i}\right)$ be a higher derivation of rank $s<\infty$ of $R$. If $d \mathcal{O} \subset \mathcal{O}$ and $d(\Omega(\mathcal{O}, R)) \subset R(\mathcal{O}, R)$, then for any $x \in R(\mathcal{O}, R)^{*}$, there exists a common element $y \in \mathcal{O}$ such that $y d_{i}(x) \in \mathbb{R}(\mathcal{O}, R)$ for all $i$.

CoROllaRY 2. Under the assumptions of Theorem $3, d k \subset k$ implies $d(R(k, R)) \subset R(k, R)$ and $d\left(R(k, R)^{*}\right) \subset R(k, R)^{*}$.

Proposition 3. Let $R \supset \mathcal{O}$ be two domains and let $\mathcal{O}$ contain the rational number field. Further, let $d \mathcal{O} \subset \mathcal{O}$, then, for any invertible element $x$ of $R(\mathcal{\theta}, R), d_{i}(x) \in R(\mathcal{\theta}, R)$ for all $i$.

Proof. $x \in R(\mathcal{O}, R)$ implies $x^{r} \in \mathcal{O}$ for some integer $r \geqq 1$. By the assumption, 


$$
\exp (d)\left(x^{r}\right)=[\exp (d)(x)]^{r}=\left(d_{0}(x), d_{1}(x), \cdots\right)^{r} \in \mathcal{O}^{(\infty)} .
$$

Hence $d_{0}(x)^{r} \in \mathcal{O}$, and $d_{0}(x) \in \mathcal{Q}(\mathcal{\theta}, R)$. As $x$ is an invertible element, there is a unique element $x^{-1} \in R(\theta, R)$ such that $x x^{-1}=1$. From the above discussion, $d_{0}(x) \in R(\theta, R)$. Since $d_{0}$ is a homomorphism, $d_{0}(x)$ is an invertible element of $R(\theta, R)$. Now, the $t$ th component of $\left(d_{0}(x), d_{1}(x), \cdots, d_{t-1}(x), \cdots\right)^{r}$ is

$$
\left(\begin{array}{l}
r \\
1
\end{array}\right) d_{0}(x)^{r-1} d_{t}(x)+\Delta\left(d_{0}(x), d_{1}(x), \cdots, d_{t-1}(x)\right)
$$

where $\Delta\left(d_{0}(x), d_{1}(x), \cdots, d_{t-1}(x)\right)$ is a polynomial in the $d_{0}(x)$, $d_{1}(x), \cdots, d_{t-1}(x)$ with rational coefficients. Assume that, for $i \leqq N$ $-1, d_{i}(x) \in \mathbb{R}(\mathcal{O}, R)$. Then, $\left(\begin{array}{l}r \\ 1\end{array}\right) d_{0}(x)^{r-1} d_{t}(x) \in \mathcal{R}(\mathcal{O}, R)$. Since $\mathcal{O}$ contains rational numbers and $d_{0}(x)^{-1} \in R(\mathcal{\theta}, R), d_{t}(x) \in R(\mathcal{O}, R)$. Hence for all $j \geqq 0, d_{j}(x) \in R(\theta, R)$. This completes the proof.

Lemma 1. A ssume that a quasi-local ring $(0, m)$ is dominated by another quasi-local ring $(R, M)$. Then $\mathbb{R}(\mathcal{O}, R)$ is a quasi-local ring.

Proof. It is sufficient to prove that $m=M \cap R(\mathcal{O}, R)$ is the unique maximal ideal of $R(\theta, R)$. Assume that $x \in R(\theta, R)-m$. Then, for some in teger $r \geqq 1, x^{r} \in \mathcal{O}$ and $x^{r} \notin m$. Hence $x^{-r} \in \mathcal{O}$ and $x^{-1} \in R$. Thus $x^{-1} \in R(\theta, R)$.

TheOREM 5. Let $(R, M)$ and $(\mathcal{O}, m)$ be two quasi-local rings and let $d=\left(1, d_{1}, d_{2}, \cdots\right)$ be a higher derivation of rank $\infty$ of the total quotient ring of $R$ such that doCO. Suppose that $R$ is integral over $\mathcal{O}$ and $\mathcal{O}$ is dominated by $R$. Then $d m \subset m$ implies $d M \subset M$.

Proof. By virtue of Theorem 2, there exists a prime ideal $M^{\prime}$ of $R$ such that $M^{\prime} \cap \mathcal{O}=m$ and $d M^{\prime} \subset M^{\prime}$. On the other hand, $M \supset M^{\prime}$.

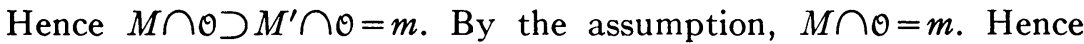
$M=M^{\prime}$. Thus we have $d M \subset M$.

CoRollary 1. Let $(R, M)$ and $(\mathcal{O}, m)$ be two quasi-local rings and let $d=\left(1, d_{1}, d_{2}, \cdots\right)$ be a higher derivation of rank $\infty$ of the total quotient ring of $R$ such that doCO. Assume that $\mathcal{O}$ is dominated by $R$. Then $d(M \cap \Re(\mathcal{O}, R)) \subset M \cap \Re(\mathcal{O}, R)$.

Proof. The first half is the consequence of Lemma 1, and the second half is proved the same way as Theorem 5.

CoROllaRY 2. Under the assumption of Corollary 1 , let $\theta$ contain the rational number field. Then $d(\Re(\theta, R)) \subset \Re(\theta, R)$. 
Proof. It follows obviously from Proposition 3 and Corollary 1.

Acknowledgement. The author wishes to express his hearty thanks to Professor Y. Nakai for his valuable suggestions.

\section{REFERENCES}

1. P. Ribenboim, Higher derivations of rings, Queen's Math. Preprints \#1969-20, Queen's University, Kingston, Ontario, Canada.

2. A. Seidenberg, Derivations and integral closure, Pacific J. Math. 16 (1966), 167173. MR $32 \# 5686$.

3. - Differential ideals in rings of finitely generated type, Amer. J. Math. 89 (1967), 22-42. MR 35 \#2902.

4. N. Jacobson, Lectures in abstract algebra. Vol. 3: Theory of fields and Galois theory, Van Nostrand, Princeton, N. J., 1964. MR 30 \#3087.

Oita University, Oita, Japan 